ACTA SCIENTIFIC PHARMACEUTICAL SCIENCES (ISSN: 2581-5423)

Volume 3 Issue 10 October 2019

Short Communication

\title{
Mushrooms as Plastic Eaters
}

\section{Charu Gupta* and Dhan Prakash}

Amity Institute for Herbal Research and Studies, Amity University, Uttar Pradesh, India

*Corresponding Author: Charu Gupta, Amity Institute for Herbal Research and Studies, Amity University, Uttar Pradesh, India.

Received: September 05, 2019; Published: September 12, 2019

DOI: $10.31080 /$ ASPS.2019.03.0397

Fungi are remarkable in the diversity of substrates they use as food sources. This has led to a growing list of species being used for the bioremediation of environments contaminated by pollutants such as oil spills and toxic chemicals (e.g. TNT, sarin nerve gas, pesticides). Nevertheless, their use for cleaning up sites contaminated by acidic radioactive waste is only just starting to be realized, following the discovery of a fungus (Rhodotorula taiwanensis) that not only grows in extremely acidic conditions $(\mathrm{pH} 2.3)$ but can also tolerate such high levels of gamma radiation that it is one of the most radiation-resistant organisms on Earth. Its genome has recently been sequenced to decipher the genomic machinery enabling it to survive and to determine how this can be utilized to develop new ways to clean up soils contaminated by radioactive waste. Similar approaches are also proposed in a recent study that has discovered a fungus (Aspergillus tubingensis) capable of breaking down plastics such as polyester polyurethane (used in a wide diversity of products such as refrigerator insulation and synthetic leather) in weeks rather than years. This potential of mushrooms can be exploited as an effective tool to combat plastic pollution and their disposal.

Plastic pollution is now a menace to the society and the environment that has to be dealt with immediate action, before it completely overwhelms us.

It is estimated that world have over 150 million tons of plastic in the oceans and by 2050, there could be more plastic than fish. Plastic is infamous for its endurance and resistance to regular decomposition. Thus it is a need of an hour to discover for a faster, effective, ecofriendly and a natural method of plastic disposal.
There are numerous mushrooms species that are gifted of consuming plastic as their chief carbon source, such as the common edible oyster mushroom. In the Amazon rainforest, a rare species of mushroom Pestalotiopsis microspore has been discovered that is capable of surviving on plastic only. It ingests polyurethane, the basic component in plastic goods, and changes it to organic matter. This mushroom can also survive without oxygen, which advocates their huge prospective for nourishing on, and thus scrubbing even at the extremity of landfills. This unusual mushroom could help as the principal of municipal waste treatment centers. Instead of discarding and burning the refuse, these mushrooms could be used to decompose the huge quantities of plastic. In this way, they could even be developed and used in composting systems at home. More importantly, some of them can remove plastic and end in edible mushrooms that can be consumed safely as food [1].

Recently, some scientists have developed a system called as the Fungi Mutarium. This is mainly a mini-garden that permits growing two fungi strains- Schizophyllum commune (Split gill mushrooms) and Pleurotus ostreatus (Oyster mushrooms) simultaneously. Both of these are the edible mushrooms and can also 'eat' massive amounts of plastic.

The process begins with sterilization of the target plastic material under UV rays by keeping it in an activation chamber where UV light sterilizes the material and initiates the degradation process. The plastic is then employed in the growth sphere where it stands in an egg-shaped case prepared from agar (a gelatinous material used to culture samples). These cases are named FUs. The thinned mycelium is added to the FUs that gradually start consuming the plastic, and grows into a cottony mushroom-like stuff. The incuba- 
tion period is for several months during which the mycelium culture degrades and consumes plastic. Research is going on hastening the biodegradation process by optimizing culture conditions.

Under controlled conditions, this mushroom mycelium takes a few weeks to start decomposition process, and within a few months, the plastic is completely broken down, leaving a white bulbous mushroom as residue. These residues can be composted and recycled to soil at a much faster rate than that for plastic, which is projected to take around 400 years to decompose on its own [2].

Since mycelium are natural decomposers, they help to break down dead trees and return them to the soil, thus turning them toward breaking down plastic, even though plastic is not an organic material. Thus mushrooms ascertain themselves to be gifted of enchanted once again [2].

In a recent study, the researchers first sterilized the test sample of plastic under UV light, before starting the degradation process. The supposed plastic sample was then positioned in a little cuplike section with a small case of agar, which is a jelly-like substance made from red algae. The fungal mycelium was then added to these cups, and allowed to grow in the shell, eventually consuming the plastic and turning into a cottony mushroom-like structure.

This complete process takes about two months, but the investigators are still working on a method to speed along the process. Additionally, one can add flavours to these cups for increasing their palatability.

The researchers themselves consumed these mushrooms and no negative effects were observed except that the finished product tasted sweet with an odor of anise or licorice. Still scientific validation is required to confirm their non-toxicity and safety. After the complete scientific validation, our plastic pollution and food crisis problem can be solved in one hit.

The researchers believe that the digestion process can be enhanced by optimization of the culture conditions. This research has open up an idea of setting small mushroom farm in any rural area that can be used for combating food crisis \& hunger along with dealing with plastic pollution.
Another example of a biodegrading fungus is Aspergillus tubingensis that was discovered in a landfill in Islamabad, Pakistan. Studies showed that this fungus is also proficient of inhabiting polyester polyurethane (PU) and break down it into smaller pieces only within a small time of two months. The fungus possesses the ability to propagate directly on the plastic surface, as it breakdown the chemical bonds between the plastic molecules through the secretion of a unique extracellular enzyme.

The research team has discovered around 50 more species since 2017 and is currently working on optimizing the culture conditions of temperature and environment for each strain of fungus for exploiting their maximum potential on large scale to the real application of plastic waste.

The studies have also established that the two white rot varieties of fungus like Pleurotus ostratus and Trametes versicolor have a favorable effect on soil and wastewater, eliminating pesticides, colorants and explosive fragments. The Trichoderma species has been acknowledged as a stimulant for generating biofuels through its transformation of agricultural waste into ethanol sugars. Fungal mycelium is also famous for designers and architects concerned in discovering viable substitutes for polystyrene foam, leather and several building materials [3].

The few challenges for industrialization are the sensitivity of fungus to infection from bacteria which could turn it into a destructive mold. Thus decontamination of the materials, developing fungal resistant strains, that are robust, and fast-multiplying for commercial processes are some of the areas of future research [4].

This research holds prospective in solving another problem of world malnutrition as according to the estimates, around 100 million people in the world starve every day and this mushroom provides an opportunity to convert waste to gem.

The other benefits of this fungus are that they can bare the mushroom's ability to eliminate contaminants from soil and to permit the alteration of waste into biofuels. These remarkable features of mushrooms make them a potential candidate for combating world pollution and food crisis. Thus it can be safely concluded that there is a vast potential for mushrooms and fungi in areas as diverse as biofuels, medicines and other unique materials. 


\section{Bibliography}

1. D'Mello G. "This Plastic-Eating Mushroom Can Solve Our Plastic Pollution By Turning Waste Into Food" (2019).

2. Denhof S. "Pestalotiopsis Microspora: The Mushroom That Eats Plastic" (2019).

3. Pricop L. "Plastic-eating mushrooms are the new superheroes in combating the growing waste crisis" (2018).

4. Hildebrandt E. " 50 New Plastic-Eating Mushrooms Have Been Discovered in Past Two Years" (2019).

Volume 3 Issue 10 October 2019

(C) All rights are reserved by Charu Gupta and Dhan

Prakash. 